\title{
Research Paper: Factor Structure and Psychometric Properties of the Multidimensional Body-self Relations Questionnaire (MBSRQ) in Female Iranian University Students
}

Yousef Khodabandelou ${ }^{*} \mathrm{O}$, Jalil Fat'h-Abadi $^{2}$, Negin $^{\prime}$ Motamed-Yeganeh ${ }^{3}$, Sara Yadollahi $^{4}$

1. Department of Clinical Psychology, Faculty of Psychology and Educational Sciences, Alameh Tabataba'i University, Tehran, Iran.

2. Department of Clinical Psychology, Faculty of Psychology and Educational Sciences, Shahid Beheshti University, Tehran, Iran

3. Department of Clinical Psychology, Faculty of Psychology and Educational Sciences, University of Tehran, Tehran, Iran.

4. Department of Clinical Psychology and Psychometrics, Faculty of Psychology and Social Sciences, Tehran-Markaz branch, Islamic Azad University, Tehran, Iran.

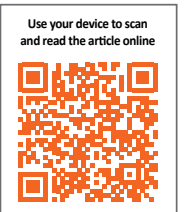

citation: Khodabandelou, Y., Fat'h-Abadi, J., Motamed-Yeganeh, N., \& Yadollahi, S. (2019). Factor Structure and Psychometric Properties of the Multidimensional Body-self Relations Questionnaire (MBSRQ) in Female Iranian University Students. Journal of Practice in Clinical Psychology, 7(3), 187-196. http://dx.doi.org/10.32598/jpcp.7.3.187

http://dx.doi.org/10.32598/jpcp.7.3.187

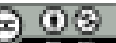

Article info:

Received: 29 Nov 2019

Accepted: 09 Apr 2019

Available Online: 01 Jul 2019

\section{Keywords:}

Multidimensional Body-Self Relations Questionnaire, Psychometric properties, Reliability, Validity

\section{ABSTRACT}

Objective: Body image is a complex and multifaceted construct encompassing at least perceptual, affective, cognitive, and possibly behavioral aspects of body experience. In the current Iranian society, increasingly the major focus is on the body's appearance, in particular on body shape and weight. Current societal standards for female beauty excessively emphasize the desirability of thinness; an ideal accepted by most females, but impossible for most of them to achieve. These body image concerns can be measured using Multidimensional BodySelf Relations Questionnaire (MBSRQ-AS). The present study aimed at testing the factorial structure and determining the psychometric properties of the MBSRQ in female Iranian university students.

Methods: The MBSRQ-AS was administered to 496 participants aged 16 to 39 selected using multistage cluster sampling method among the female students of Tehran University from 70 fields of study.

Results: Exploratory factor analysis was used to determine the construct validity of the questionnaire. Principal component analysis using Varimax rotations resulted in a five-factor structure $(\mathrm{KMO}=0.56$, Chi-square $=5419.80, \mathrm{df}=561)$ including: appearance orientation, appearance evaluation, overweight preoccupation, self-classified weight and body areas satisfaction. confirmatory factor analysis showed that the extracted model had a good fit to the data $(\mathrm{RMSEA}=0.064, \mathrm{NFI}=0.88, \mathrm{CFI}=0.91)$. Reliability analysis of total score of MBSRQAS and its subscales suggested good internal consistency (Cronbach's alpha coefficient for total scale $=0.83$ ).

Conclusion: In conclusion, the results of the current study provided strong evidence for reliability and validity of MBSRQ-AS as a multidimensional tool to measure body image among Iranian students.

\section{* Corresponding Author:}

Yousef Khodabandelou, PhD

Address: Department of Clinical Psychology, Faculty of Psychology and Educational Sciences, Alameh Tabataba'i University, Tehran, Iran.

Tel: + 98 (939) 393474632

E-mail:y.khodabande@gmail.com 


\section{Highlights}

- Body image is a complex concept which involves perceptual, affective, cognitive, and behavioral aspects of body experience.

- In contemporary Iranian society, people and especially women are more concerned about their body appearance, in particular, body shape and weight.

- The multidimensional body-self relations questionnaire is a tool to measure the body image.

- Our study results strongly support the reliability and validity of this tool for measuring body image among Iranian students.

\section{Plain Language Summary}

Body image is a complex concept involving perceptual, affective, cognitive, and behavioral aspects of body experience. Nowadays, Iranian have more and more got concerned about their body image. Especially Iranian women have become obsessed with their weight and appearances. One of the tools to measure this obsession among people is the multidimensional body-self relations questionnaire. In this study, we examined the reliability of this test in a sample of Iranian female students. The results provided substantial evidence to support the reliability and validity of this questionnaire as a multidimensional tool for measuring body image among Iranian students.

\section{Introduction}

Body image is a "multifaceted psycho$\mathrm{B}$ logical experience of embodiment" that consists of evaluative thoughts and beliefs towards one's own physical appearance that it leads to special feelings and behaviors (Cash, 2004). Cognitive-behavioral theory of body image (Cash, 2002) believes that the core beliefs related to appearance, determines the amount of investment on the appearance to a large extent (Cash, Melnyk, \& Hrabosky, 2004).

Females of all ages, especially teenagers, are vulnerable to disturbance in body image; Negative body image in women is a common phenomenon with high prevalence (Kops, Bessel, Knauth et al., 2018; Garrusi, \& Baneshi, 2017). "normative discontent" is a phrase used by researchers to naming women's concern about their physical appearance that it points to this subject that negative body image affects almost all women at some level (Breannan, Lalonde, \& Bain, 2010; Tiggemann \& Slater, 2004). It has been shown that females have a more negative body image than males (Breannan, Lalonde, \& Bain, 2010), and females of all ages and sizes suffers from negative body image and it is also common in other societies with a high socioeconomic status (Swami, Frederick, Aavik et al., 2010).
According to Iranian population-based surveys, negative body image is increasing (Butters \& Cash, 1987). It seems that negative body image is more related to thoughts about appearance than being associated with physical reality. Regardless of body mass, people are at higher risk to display negative body image if they maintain ineffective beliefs and cognitions about their physical appearance (Butters \& Cash, 1987). More than $43 \%$ college women want to lose weight, while many of them have healthy body mass index (Fayet, Petocz, \& Samman, 2012) Furthermore, negative body image also is certainly common in others populations. For example, negative body image is one of the most common and prominent symptoms in clinical population such as people with eating disorders (McLean \& Paxton, 2019). Negative body image is also associated with the chronic use of appearance and performance enhancing drugs (Hildebrandt, Alfano, \& Langenbucher, 2010), and it's a predictor factor for depression among females (Murray, Rieger, \& Byrne, 2018).

Since body image is among the most controversial construct in psychology today, its assessment has become an important issue as well. The Multidimensional Body-Self Relations Questionnaire -Appearance Scales (MBSRQAS by Cash, 2000, 2015) were designed to evaluate the different dimensions of body image. The MBSRQ-AS is a self-report inventory that consists of 34 items and five subscales: Appearance Orientation (AO), Appearance 
Evaluation (AE), Overweight Preoccupation (OP), Body Areas Satisfaction Scale (BASS), and Self-Classified Weight (SCW). The MBSRQ-AS has been used in various researches with clinical and nonclinical populations (e.g. Cash, 2015). The MBSRQ and MBSRQ-AS have been translated into different languages in several researches. These researches have used different methods and have achieved different results. Factor structure and psychometric properties of the Malaysian Malay version of the MBSRQ-AS were investigated with 18 to 64 years participants. Results demonstrated that this measure has a good reliability and validity in Malaysian Malay adults. In another research, factor structure and psychometric properties of the French version of the MBSRQAS was investigated with 18 to 61 years participants. When only two main factors of the questionnaire $(\mathrm{AE}$ and $\mathrm{AO}$ ) were used in the exploratory factor analysis, explained $42.3 \%$ of the variance (Untas, Koleck, Rascle, $\&$ Borteyrou, 2009). Confirmatory factor analysis of the German version of the MBSRQ-AS was investigated by Vossbeck-Elsebusch, Waldorf, Legenbauer et al., (2014). Results showed an goodness of fit for a four-factor model (AO, AE, BASS, and OP). The Spanish adaptation of the questionnaire was investigated by Marcoa, Perpiná, Roncero \& Botellac (2017) with 18 to 61 and 12 to 14 years participants. When the researchers used confirmatory factor analysis, results showed a excellent goodness of fit for a five-factor model. The Greek version of the questionnaire with 15 to 19 years participants was investigated by Argyrides \& Kkeli (2013). When only two main factors of the questionnaire (AE and $\mathrm{AO}$ ) were used in the exploratory factor analysis, explained $41.44 \%$ of the variance.

Although there is a lack of evidence regarding evaluating body image by a valid Persian instrument, MBSRQ-AS hasn't been validated in Persian sample yet and this is the first study that the reliability and validity of the Persian version of MBSRQ-AS be tested among a group of female Iranian students. Therefore, the necessity and importance of the focus on technical features of MBSRQAS can be explained among a group of Iranian students. To measure body image structures, accessing to a valid and reliable instrument is inevitable in different cultural realms. The aim of the present study was to confirm the factorial structure of the MBSRQ-AS in a representative sample consisting of female Iranian students and analyze its psychometric properties.

\section{Methods}

\section{Participants}

Participants were 496 female students from University of Tehran with average age of 18.21 years $(\mathrm{SD}=2.51)$, ranging from 16 to 39 . Descriptive statistics showed that $89.5 \%$ of the subjects were bachelor's degree, $7.7 \%$ master's degree and $2.8 \%$ doctoral degree students. In terms of marital status, $92.5 \%$ of the participants were single and $7.5 \%$ married. The students were majoring in 17 fields of study (e.g. Physics, Biology, Mathematics, Psychology, etc.).

\section{Instrument}

The Multidimensional Body-Self Relations Questionnaire- Appearance Scales (MBSRQ-AS) (Cash, 2000; Cash, 2015) was used. The MBSRQ is a self-report inventory that assesses peoples' attitudes toward the different aspects of body image intended to be used for adults and adolescents (15 years or older). Two forms of the MBSRQ are available. The full, 69-item version consists of 10 subscales: Evaluation and Orientation vis-à-vis Appearance, Fitness, and Health/Illness, plus OP, SCW, and the BASS. Internal consistency for the subscales of the MBSRQ ranged from 0.67 to 0.85 for males and 0.71 to 0.86 for females. The short form of MBSRQ-AS is a 34-item measure that consists of five subscales: $\mathrm{AE}, \mathrm{AO}$, $\mathrm{OP}, \mathrm{SCW}$, and the BASS. Internal consistencies of the subscales ranged from 0.76 to 0.86 and test-retest reliabilities ranged from 0.75 to 0.93 . Convergent validity was also confirmed. The current study used the short version of MBSRQ.

Respondents rate their level of agreement with statements such as, "I like my looks just the way they are", or "I am physically unattractive", on a 5-point Likert type scale, ranging from (1) "Definitely Disagree" to (5) "Definitely Agree". High scores on this measure indicate increased dissatisfaction with general body image.

\section{Procedure}

First, the MBSRQ was translated and adapted into Persian. Forward and backward translation procedures were used (Marsella \& Leong, 1995). First, a professional translator translated the MBSRQ from English to Persian, and then a back translation was applied by a second professional translator. Afterwards, both interpreters discussed the differences between English and Persian versions. Through an iterative review process, these differences were minimized. Accordingly, the se- 
quence of the original semantic version was carefully examined. Finally, the cultural adaptation and content validity of the scale was read and approved by several faculty members.

Second, Krejcie and Morgan's Table was used to determine the optimal sample size (384 participants were suggested with $0.5 \%$ margin of error and $95 \%$ level of confidence). Multistage cluster sampling method was used to collect the data. The entire population (population of the entire university) was divided into different cluster (faculties). Then five faculties were selected through simple random sampling and the participants were randomly selected from each cluster. Data were collected on the campus of the faculties; 496 participants were asked to take part in the study. No payment was offered and participation was voluntary. All of the subjects were informed that their responses would remain anonymous. After providing their consent they were asked to complete the measures.

\section{Data analysis}

To examine the internal consistency of the scores and factors, Cronbach's alpha was computed for total scale and the extracted subscales. In order to determine the construct validity, exploratory factor analysis (principal component analysis method) was used to extract the underlying components of the scale and then the extracted model was examined using confirmatory factor analysis. Such a two-step method was developed since the underlying structure of the Persian version questionnaire was not investigated on Iranian students before and the original presumed structure of the English version was not applicable. Hence, the test structure was investigated using an exploratory method and the extracted structure was then subject to confirmatory techniques.

\section{Results}

\section{Reliability of the MBSRQ}

One of the assumptions of factor analysis is adequacy of the sample size. Thus, at this stage we marked the KMO as a sampling adequacy assessment. KaiserMeyer-Olkin measure of sampling adequacy calculated before performing exploratory factor analysis, and was found to equal 0.85 . Given that, when $\mathrm{KMO}$ is between 0.80 to 0.90 , it can be represented that the sample size is sufficient and appropriate (Hooman, 2001). Another assumption of factor analysis is known as a multivariate normal distribution (spherical). According to the chi-square the following table indicating multivariate normal distribution status and significance of spherical (Table 1 and Figure 1).

\section{Validation of the MBERQ}

One of the assumptions of factor analysis is adequacy of the sample size. Thus, at this stage we marked the KMO as a sampling adequacy assessment. KaiserMeyer-Olkin measure of sampling adequacy calculated before performing exploratory factor analysis, and was found to equal 0.85 . Given that, when $\mathrm{KMO}$ is between 0.80 to 0.90 , it can be represented that the sample size is sufficient and appropriate (Hooman, 2001). Another

Table 1. Kaiser-Meyer-Olkin Measure of Sampling Adequency and Bartlett's test

\begin{tabular}{cccccc}
\hline Interpretation & Sphericity Test & Approx. Chi-square & DF & Sig. & Sphericity Test \\
\hline $\begin{array}{c}\text { Perfect sphericity (the multivariate normal distribu- } \\
\text { tion is obtained) }\end{array}$ & Bartlett's test & 5419.798 & 561 & 0.001 & Bartlett's test \\
\hline
\end{tabular}

Table 2. Total variance explained table

\begin{tabular}{|c|c|c|c|}
\hline Component & Eigen Values & Variance (\%) & Cumulative (\%) \\
\hline 1 & 5.607 & 20.766 & 20.766 \\
\hline 2 & 3.866 & 14.320 & 35.086 \\
\hline 3 & 1.616 & 5.985 & 41.072 \\
\hline 4 & 1.278 & 4.734 & 45.806 \\
\hline 5 & 1.165 & 4.315 & 50.121 \\
\hline
\end{tabular}




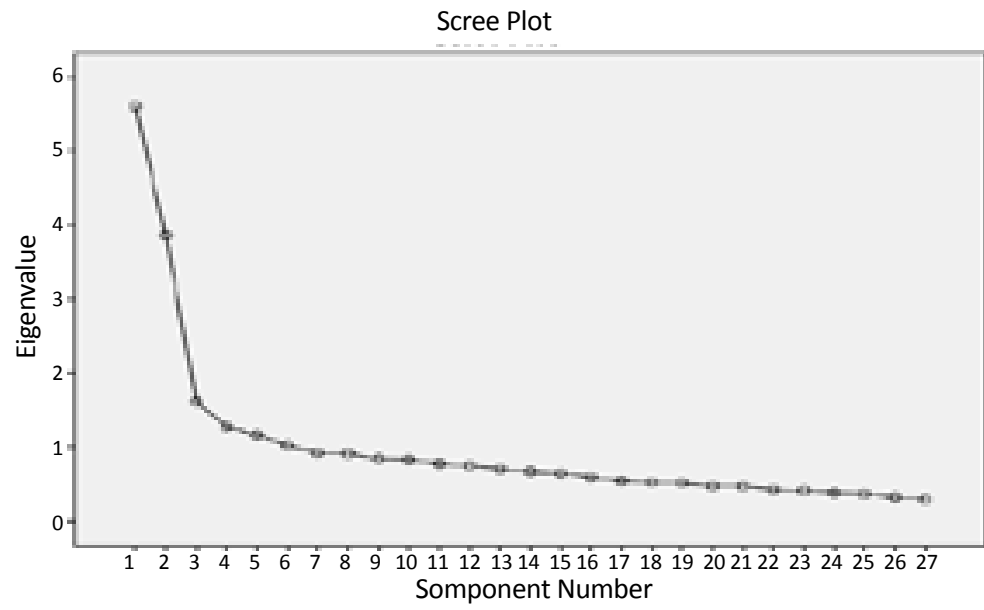

Figure 1. Scree plot

Table 3. Results of exploratory factor analysis, extracted components and factor loading of each item

\begin{tabular}{cccccc}
\hline Factor & Load Factor & Extraction & Factor & Load Factor & Extraction \\
\hline 6 & 0.64 & 0.41 & 7 & 0.70 & 0.49 \\
8 & 0.61 & 0.37 & 10 & 0.68 & 0.46 \\
13 & 0.59 & 0.35 & 17 & 0.64 & 0.38 \\
21 & 0.65 & 0.42 & & & \\
\hline
\end{tabular}

First factor: Appearance evaluation, $\mathrm{a}=0.761$

\begin{tabular}{cccccc}
\hline Factor & Load Factor & Extraction & Factor & Load Factor & Extraction \\
\hline 28 & 0.71 & 0.51 & 29 & 0.75 & 0.56 \\
30 & 0.65 & 0.42 & 31 & 0.60 & 0.36 \\
32 & 0.78 & 0.61 & 33 & 0.51 & 0.26 \\
\hline
\end{tabular}

Second factor:Appearance Orientation, $\mathrm{a}=0.758$

\begin{tabular}{cccccc}
\hline Factor & Load Factor & Extraction & Factor & Load Factor & Extraction \\
\hline 5 & 0.74 & 0.54 & 9 & 0.64 & 0.411 \\
26 & 0.82 & 0.67 & 27 & 0.55 & 0.30 \\
34 & 0.78 & 0.61 & & & \\
\hline
\end{tabular}

Third factor: Body areas satisfaction, $\mathrm{a}=0.739$

\begin{tabular}{cccccc}
\hline Factor & Load Factor & Extraction & Factor & Load Factor & Extraction \\
\hline 3 & 0.58 & 0.33 & 12 & 0.72 & 0.52 \\
18 & 0.81 & 0.65 & 19 & 0.82 & 0.68 \\
\hline
\end{tabular}

Forth factor: Overweight preoccupation, $\mathrm{a}=0.722$

\begin{tabular}{cccccc}
\hline Factor & Load Factor & Extraction & Factor & Load Factor & Extraction \\
\hline 1 & 0.77 & 0.60 & 2 & 0.74 & 0.55 \\
14 & 0.70 & 0.49 & 29 & 0.52 & 0.27 \\
\hline
\end{tabular}

Fifth factor: Self-classified weight, $\mathrm{a}=0.618$ 


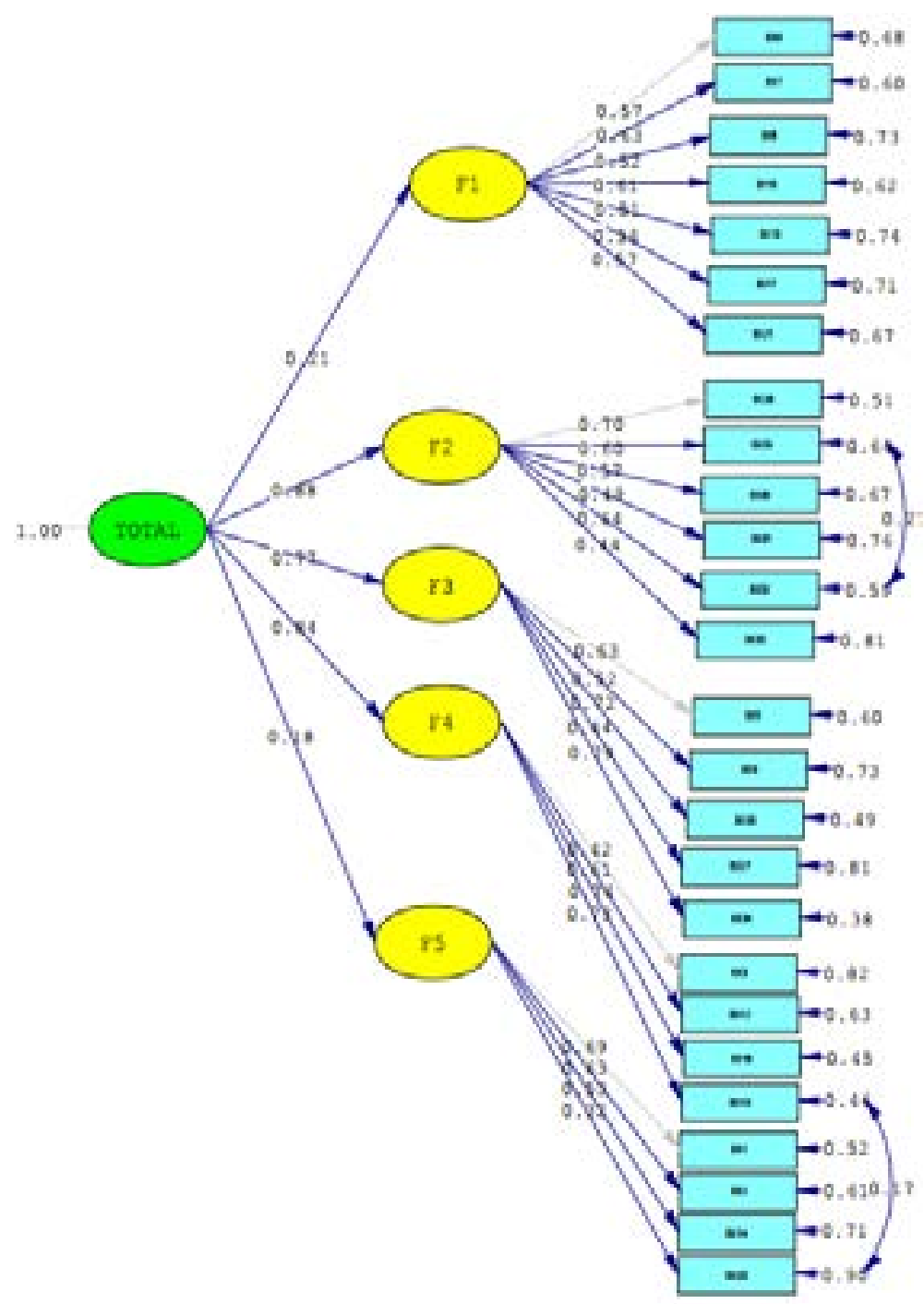

Figure 2. Confirmatory factor analysis

assumption of factor analysis is known as a multivariate normal distribution (spherical). According to the chi-square the following table indicating multivariate normal distribution status and significance of spherical.

The results show that the sample and correlation matrix for this analysis were appropriate. Calculating the amount of material communality in the set of 26 questions showed that the lowest communalities are equal with 0.381 and 0.385 and they belong to questions 6 and 13. The most communalities are equal to 0.67 and 0.72 and they belong to questions 18 and 19 .

After performing factor analysis and extracted several components and comparing them with the theoretical structure and scale of the theory, finally five components were extracted and for extracting these components, the multiple dimensions of correlation and varimax rotation methods were used. The results showed that the sample and matrix correlation for these analyses were appropriate. Results of principal components analysis suggest that the Eigenvalues of five components are greater than 1 , and 50.12 percent of the total variance was explained by these variables (Table 2).

Since the definition of Eigenvalues is total load factor, so in the process of identifying and extracting the components, the specific values of more than 1 is emphasized and the Scree plot is present in the following table.

With reference to Scree plot and total variance explained Table 2, maximum 5 components were extracted based on columns of Eigen values. The first component explained that the variance is high, and the amount of it in the Scree plot is more visible. 
After exploratory factor analysis process, it was found that five of the rotation factor analysis, extract, and in fact, the body image questionnaire consists of five components. The first component has the most variable on it. The following table is Findings of the load by analyzing the principle components on which factor is observable.

Then, the confirmatory factor analysis was used to confirm the components which are extracted from the exploratory factor analysis from the MBSRQ-AS questionnaires in Iranian students. Following the Bentler and Bonett (1980) criteria for the fit indices: Comparative Fit Index and Root Mean-Square Error of Approximation, the proposed structure of the questionnaire was confirmed. Hu and Bentler (1999) suggested that a comparative fit index of at least .90 and a root mean-square error of approximation of less than .06 would together indicate a good fit. Considering the type of the variables (ordered, categorical and non-normal), as the estimation method in EQS, maximum likelihood Satorra-Bentler corrected standard errors, polychoric correlations, and test statistics were used (Finney \& DiStefano, 2006).

In this study the confirmatory factor analysis was conducted with using LISREL software on five-factor model. Five-factor model consists of variables of $\mathrm{AO}, \mathrm{AE}$, OP, SCW and BASS scales (Table 3).

Confirmatory factor analysis's results in a sample of Iranian students empirically supported the five-factor structure of the questionnaire. The results of goodness of fit statistics for the five-factor model in the case of Iranian students accordingly to the Hu \& Bentler (1999) proposed, are included Normal Theory Weighted Least Squares Chi-Square $=883.39$, Chi-Square difference with 1 Degree of freedom=67.55, Root Mean Square Error of Approximation=0.064, Goodness of Fit Index $=0.88$, Comparative Fit Index Confirmatory factor analysis's results in a sample of Iranian students empirically supported the five-factor structure of the questionnaire. The results of goodness of fit statistics for the five-factor model in the case of Iranian students accordingly to the $\mathrm{Hu} \&$ Bentler (1999) proposed, are included Normal Theory Weighted Least Squares ChiSquare $=883.39$, Chi-Square Difference with 1 Degree of Freedom $=67.55$, Root Mean Square Error of Approximation $=0.064$, Goodness of Fit Index $=0.88$, Comparative Fit Index $=0.91$, Normed Fit Index $=0.88$, Adjusted Goodness of Fit Index $=0.85$, Parsimonious Normed Fit Index $=0.707$ and Parsimonious Comparative Fit Index $=$ 0.724 .All indicators suggest that the mentioned model has a good fit to the data (Kline, 2011) (Figure 2).

\section{Discussion}

In this study, the reliability and validity of the psychometric characteristics of the English version of the Multidimensional Body-Self Relations Questionnaire -Appearance Scales (MBSRQ-AS) were tested for the first time among a group of Iranian females. The empirical findings from the exploratory factor analysis supported the validity of the factors. With emphasis on current research findings that pay off on the reliability and validity of the Multidimensional body self-relations questioner and using Principle components with Varimax rotation; it can be said that it has a factorial validity of the scale with body image.

Results of this study has confirmed the factorial structure of body image construct proposed by Cash (2000, 2015) with appropriate fit indices. AO, AE, BASS, OP, and SCW are the factors that extract from this research. The five subscales of the MBSRQ-AS generally have good psychometric qualities. The total internal consistency is 0.83 . The scores on the five subscales showed adequate internal consistency, and these results are similar to those from other studies (Cash, 2000; Untas, Koleck, Rascle, \& Borteyrou, 2009; Vossbeck-Elsebusch et al., 2014). Various studies have used the MBSRQ-AS, for example Swami et al., (2019) demonstrated that this measure has a good reliability and validity in Malaysian Malay adults.

It worth considering that 8 out of 34 items had to be deleted because of their negative effects on reliability which could reflect the properties of the Persian sample. In most studies that determining psychometric properties of various versions of MBSRQ-AS, considerable range of ages in the sample could be noted. However, female students were target population of the present study. So the observed differences in results could be explained by the fact that body image is a common phenomenon especially in groups consisting of young women (Kops, Bessel, Knauth et al., 2018). Therefore, different questionnaires might be well-suited for different age groups.

Despite the deleted items which resulted in a short form of the questionnaire, findings related to the construct validity of the questionnaire were in harmony with the English version of MBSRQ-AS. Although French, Malaysian and German version of the questionnaire were found to have 2- and 4-factorial structures, respectively (Untas et al., 2009, Swami et al., 2019; \& VossbeckElsebusch et al., 2014), factorial structure of the Persian version of the questionnaire was found to be in accordance to the underlying model presumed by the designer 
of the questionnaire (Cash, 2000). It could be concluded that Persian sample could be fit into the theoretical framework of the construct (e.g. body image) that was conceptualized and evaluated by cash $(2000,2015)$.

As far as we know, this study is the pioneer in confirming the factorial structure of the Iranian version of the MBSRQ-AS in Iranian's sample. However, the findings of this study suffer from some limitations. First, the sample of the research included only female students. Therefore, in order to determine the potential generalizing ability of the findings to the men, additional studies are needed. Secondly, this study included only one time measuring, therefore, the test scores of MBSRQ-AS stabilization is not possible. Thus, short-term and long-term stability test of the Persian version of the MBSRQ-AS requires further studies. In the end it is recommended that for evaluating the psychometric properties of MBSRQ-AS (Stoeber \& Kersting, 2007), physiological data (Hewitt, Habke, Lee-Baggley et al., 2008) and physical data such as Body Mass Index (BMI) and waist-hip ratio (WHR) should be measured.

Despite these limitations, our findings suggest that the Persian version of the multidimensional body self-relations questioner is reliable as a new tool in the realm of body image studies to assess the psychometric accurate and psychological factor of body image.

\section{Ethical Considerations}

\section{Compliance with ethical guidelines}

All ethical principles were considered in this article. The participants were informed about the purpose of the research and its implementation stages; they were also assured about the confidentiality of their information.

\section{Funding}

This research did not receive any specific grant from funding agencies in the public, commercial, or not-forprofit sectors.

\section{Authors' contributions}

All authors contributed in preparing this article.

\section{Conflict of interest}

The authors declared no conflict of interest.

\section{References}

Argyrides, M., \& Kkeli, N. (2013). Multidimensional body-self relations questionnaire-appearance scales: Psychometric properties of the Greek version. Psychological Reports, 113(3), 885-97. [DOI:10.2466/03.07.PR0.113x29z6] [PMID]

Bentler, P. M., \& Bonett, D. G. (1980). Significance tests and goodness of fit in the analysis of covariance structures. Psychological Bulletin, 88(3), 588-606. [DOI:10.1037/0033-2909.88.3.588]

Butters, J. W., \& Cash, T. F. (1987). Cognitive-behavioral treatment of women's body-image dissatisfaction. Journal of Consulting and Clinical Psychology, 55(6), 889-97. [DOI:10.1037/0022-006X.55.6.889]

Cash, T. F. (2000). The MBSRQ users' manual. 3th Ed.

Cash, T. F. (2002). Cognitive behavioral perspectives on body image. In T. F. Cash \& T. Pruzinsky (Eds.), Body image: A handbook of theory, research and clinical practice (pp. 38-46). New York: Guilford Press

Cash, T. F. (2004). Body image: Past, present, and future. Body Image, 1(1), 1-5. [DOI:10.1016/S1740-1445(03)00011-1]

Cash, T. F. (2015). Multidimensional Body-Self Relations Questionnaire (MBSRQ). In T. Wade, (Eds.), Encyclopedia of Feeding and Eating Disorders (pp. 1-4). Berlin: Springer Science+Business Media. [DOI:10.1007/978-981-287-087-2_31] [PMID]

Cash, T. F., Melnyk, S. E., \& Hrabosky, J. I. (2004). The assessment of body image investment: An extensive revision of the Appearance Schemas Inventory. International Journal of Eating Disorders, 35(3), 305-16. [DOI:10.1002/eat.10264] [PMID]

Fayet, F., Petocz, P., \& Samman, S. (2012). Prevalence and correlates of dieting in college women: A cross sectional study. International Journal of Women's Health, 4, 405. [DOI:10.2147/ IJWH.S33920] [PMID] [PMCID]

Finney, S. J., \& DiStefano, C. (2006). Non-normal and categorical data in structural equation modeling. In G. R. Hancock \& R. O. Mueller, (Eds.), Structural Equation Modeling: A Second Course (pp. 269-314). Charlotte: Information Age Publishing.

Garrusi, B., \& Baneshi, M. R. (2017). Body dissatisfaction among Iranian youth and adults. Cadernos de Saude Publica, 33, e00024516. [DOI:10.1590/0102-311x00024516] [PMID]

Hewitt, P. L., Habke, A. M., Lee-Baggley, D. L., Sherry, S B., \& Flett, G. L. (2008). The impact of perfectionistic selfpresentation on the cognitive, affective, and physiological experience of a clinical interview. Psychiatry, 71(2), 93-122. [DOI:10.1521/psyc.2008.71.2.93] [PMID]

Hildebrandt, T., Alfano, L., \& Langenbucher, J. W. (2010). Body image disturbance in 1000 male appearance and performance enhancing drug users. Journal of Psychiatric Research, 44(13), 841-6. [DOI:10.1016/j.jpsychires.2010.01.001] [PMID] [PMCID]

Hooman, H. A. (2010). [Multivariate data analysis in scientific research (Persian)]. Tehran: Paykefarhang.

Hu, L. T., \& Bentler, P. M. (1999). Cutoff criteria for fit indexes in covariance structure analysis: Conventional criteria versus 
new alternatives. Structural Equation Modeling: A Multidisciplinary Journal, 6(1), 1-55. [DOI:10.1080/10705519909540118]

Kline, R. B. (2010). Principles and practice of structural equation modeling. New York: Guilford Press.

Kops, N. L., Bessel, M., Knauth, D. R., Caleffi, M., \& Wendland, E. M. (2019). Body image (dis) satisfaction among low-income adult women. Clinical Nutrition, 38(3), 1317-23. [DOI:10.1016/j.clnu.2018.05.022] [PMID]

Marco, J. H., Perpiñá, C., Roncero, M., \& Botella, C. (2017). Confirmatory factor analysis and psychometric properties of the Spanish version of the Multidimensional Body-Self Relations Questionnaire-Appearance Scales in early adolescents. Body Image, 21, 15-8. [DOI:10.1016/j.bodyim.2017.01.003] [PMID]

Marsella, A. J., \& Leong, F. T. (1995). Cross-cultural issues in personality and career assessment. Journal of Career Assessment, 3(2), 202-18. [DOI:10.1177/106907279500300207]

McLean, S. A., \& Paxton, S. J. (2019). Body image in the context of eating disorders. Psychiatric Clinics, 42(1), 145-56. [DOI:10.1016/j.psc.2018.10.006] [PMID]

Mond, J., Van den Berg, P., Boutelle, K., Hannan, P., \& NeumarkSztainer, D. (2011). Obesity, body dissatisfaction, and emotional well-being in early and late adolescence: findings from the project EAT study. Journal of Adolescent Health, 48(4), 373-8. [DOI:10.1016/j.jadohealth.2010.07.022] [PMID] [PMCID]

Murray, K., Rieger, E., \& Byrne, D. (2018). Body image predictors of depressive symptoms in adolescence. Journal of Adolescence, 69, 130-9. [DOI:10.1016/j.adolescence.2018.10.002] [PMID]

Stoeber, J., \& Kersting, M. (2007). Perfectionism and aptitude test performance: Testees who strive for perfection achieve better test results. Personality and Individual Differences, 42(6), 1093-03. [DOI:10.1016/j.paid.2006.09.012]

Swami, V., Frederick, D. A., Aavik, T., Alcalay, L., Allik, J., Anderson, D., et al., (2010). The attractive female body weight and female body dissatisfaction in 26 countries across 10 world regions: Results of the international body project I Personality and Social Psychology Bulletin, 36(3), 309-25. [DOI:10.1177/0146167209359702] [PMID]

Swami, V., Todd, J., Khatib, N. A. M., Toh, E. K. L., Zahari, H. S., \& Barron, D. (2019). Dimensional structure, psychometric properties, and sex invariance of a Bahasa Malaysia (Malay) translation of the Multidimensional Body-Self Relations Questionnaire-Appearance Scales (MBSRQ-AS) in Malaysian Malay adults. Body image, 28, 81-92. [DOI:10.1016/j. bodyim.2018.12.007] [PMID]

Tiggemann, M., \& Slater, A. (2004). Thin ideals in music television: A source of social comparison and body dissatisfaction. International Journal of Eating Disorders, 35(1), 48-58. [DOI:10.1002/eat.10214] [PMID]

Untas, A., Koleck, M., Rascle, N., \& Borteyrou, X. (2009). Psychometric properties of the French adaptation of the Multidimensional Body Self Relations Questionnaire- Appearance Scales. Psychological Reports, 105(2), 461-71. [DOI:10.2466/ PR0.105.2.461-471] [PMID]

Vossbeck-Elsebusch, A. N., Waldorf, M., Legenbauer, T., Bauer, A., Cordes, M., \& Vocks, S. (2014). German version of the Multidimensional Body-Self Relations questionnaire- Ap- pearance Scales (MBSRQ-AS): Confirmatory factor analysis and validation. Body Image, 11(3), 191-200. [DOI:10.1016/j. bodyim.2014.02.002] [PMID] 
This Page Intentionally Left Blank 